University of New Hampshire

University of New Hampshire Scholars' Repository

Space Science Center

Institute for the Study of Earth, Oceans, and

Space (EOS)

1996

\title{
Hard X-ray polarimetry of solar flares with BATSE
}

\author{
Mark L. McConnell \\ University of New Hampshire - Main Campus, mark.mcconnell@unh.edu \\ D J. Forrest \\ University of New Hampshire - Main Campus \\ W T. Vestrand \\ University of New Hampshire - Main Campus \\ M Finger \\ NASA/Marshall Space Flight Center
}

Follow this and additional works at: https://scholars.unh.edu/ssc

Part of the Astrophysics and Astronomy Commons

\section{Recommended Citation}

Hard X-ray polarimetry of solar flares with BATSE McConnell, M. and Forrest, D. and Vestrand, W. T. and Finger, M., AIP Conference Proceedings, 374, 368-376 (1996), DOI:http://dx.doi.org/10.1063/1.50972

This Conference Proceeding is brought to you for free and open access by the Institute for the Study of Earth, Oceans, and Space (EOS) at University of New Hampshire Scholars' Repository. It has been accepted for inclusion in Space Science Center by an authorized administrator of University of New Hampshire Scholars' Repository. For more information, please contact Scholarly.Communication@unh.edu. 


\section{AIP $\mid$ proceedings}

\section{Hard Xray polarimetry of solar flares with BATSE}

M. McConnell, D. Forrest, W. T. Vestrand, and M. Finger

Citation: AIP Conference Proceedings 374, 368 (1996); doi: 10.1063/1.50972

View online: http://dx.doi.org/10.1063/1.50972

View Table of Contents:

http://scitation.aip.org/content/aip/proceeding/aipcp/374?ver=pdfcov

Published by the AIP Publishing

\section{Articles you may be interested in}

Xray aspects of the high energy solar physics workshop: Rapporteur Paper I

AIP Conf. Proc. 374, 519 (1996); 10.1063/1.50987

Microwave and hard Xray sources in two Xclass limb flares

AIP Conf. Proc. 374, 393 (1996); 10.1063/1.50973

Solar hard Xray albedo due to Compton scattering AIP Conf. Proc. 374, 329 (1996); 10.1063/1.50968

A correlation between 4-8 MeV gammarayline fluence and $50 \mathrm{keV}$ Xray fluence in large solar flares

AIP Conf. Proc. 294, 65 (1994); 10.1063/1.45202

Relative timing of solar prompt yray line and Xray emission produced by flare accelerated ions and electrons

AIP Conf. Proc. 170, 252 (1988); 10.1063/1.37271 


\title{
Hard X-Ray Polarimetry of Solar Flares with BATSE
}

\author{
M. McConnell ${ }^{*}$, D. Forrest*, W.T. Vestrand ${ }^{*}$ and M. Finger ${ }^{\dagger}$ \\ * University of New Hampshire, Durham, New Hampshire 03824 \\ ${ }^{\dagger}$ Marshall Space Flight Center, Huntsville, Alabama 35812
}

\begin{abstract}
We describe a technique for measuring the polarization of hard Xrays from solar flares based on the angular distribution of that portion of the fiux which is scattered off the top of the Earth's atmosphere. The scattering cross section depends not only on the scatter angle itself, but on the orientation of the scatter angle with respect to the incident polarization vector. Consequently, the distribution of the observed albedo flux will depend on the direction and the polarization properties (i.e., the level of polarization and polarization angle) of the source. Since the albedo component can represent a relatively large fraction (up to $40 \%$ ) of the direct source flux, there will generally be sufficient signal for making such a measurement. The sensitivity of this approach is therefore dictated by the effective area and the ability of a detector system to 'image' the albedo fiux. The $4 \pi$ coverage of the BATSE detectors on the Compton Gamma-Ray Observatory provides an opportunity to measure both the direct and the albedo flux from a given solar flare event. Although the BATSE design (with its large field-of-view for each detector) is not optimized for albedo polarimetry, we have nonetheless investigated the feasibility of this technique using BATSE data.
\end{abstract}

\section{INTRODUCTION}

One of the fundamental properties of electromagnetic radiation is its polarization. Although this property has been exploited extensively for astrophysical studies at longer wavelengths (e.g., radio, optical and, to some extent, $\mathrm{x}$-ray wavelengths), there have been only limited efforts to measure polarization from cosmic sources at hard X-ray energies ( $\mathrm{E}>30 \mathrm{keV}$ ).

Here we investigate how one might use the radiation which is backscattered from the Earth's atmosphere to study source polarization, a technique which we call albedo polarimetry. Based on our Monte Carlo simulations, we have estimated the BATSE sensitivity to hard X-ray polarization in solar flares and find significant sensitivity to polarization even for some moderately sized (Cand M-class) events.

The study of the albedo flux will allow us to search for polarization effects within solar flares. The observational evidence for directivity within 
solar flares requires the presence of polarization in the energetic electron bremsstrahlung emission. Polarization measurements will place important constrains on the energetic electron geometries, thus complementing the spectral measurements.

\section{POLARIZATION MEASUREMENTS OF SOLAR FLARES}

The primary goal of solar flare polarimetry is to provide a direct probe of the directivity of the electrons which are accelerated during the flare. Searches for electron anisotropies in solar flares have been motivated by the clues that it can provide about electron acceleration and transport. The usual method for assaying the anisotropy is to search for evidence of directivity in the flare radiation. Until the mid 1980's all the directivity measurements were performed at energies below $100 \mathrm{keV}$. The problem at these energies is that Compton backscattering of X-rays from the solar atmosphere tends to mask the primary radiation pattern.

The large sample of flares detected at energies greater than $300 \mathrm{keV}$ by SMM GRS allowed, for the first time, a statistical search for directivity at high energies. The greatly reduced Compton reflectivity and increased directionality of the bremsstrahlung cross-section make relatively strong radiation anisotropies a possibility at these energies. The procedure used in these statistical studies was to search for disk center-to-limb variations in flare frequency and spectra. Statistically significant variations from the predictions for isotropic emission are then attributed to radiation anisotropy. Using this technique, analysis of high-energy observations by SMM GRS has provided the first relatively strong evidence for directed emission from flares (1). Data from an independent set of flares detected by instruments on the Venera 13 and 14 spacecraft has also been used to present evidence for radiation anisotropies at hard $\mathrm{x}$-ray energies (2). In this case, the positional spectral variation has also been attributed to bremsstrahlung directivity.

Another promising method for studying the directivity in individual flares is the stereoscopic technique (3), which compares simultaneous observations made on two spacecraft that view the flare from different angles. Combined observations from spectrometers aboard the PVO and ISEE-3 satellites have been used to produce stereoscopic observations of 39 flares that occurred between 1978 and 1980 (4). While the range of measured flux ratios is consistent with the statistical studies (5), the deviations of the ratio from unity show no clear correlation with increasing difference in viewing angles. It has therefore been argued that directivity conclusions based on stereoscopic observations for single flares are not yet possible because of the large error bars (6).

Quantifying the magnitude of the directivity from these statistical measurements is difficult. For example, one needs to know the size-frequency distribution for flares as well as the form of the electron distribution to derive the predicted limb fraction (7). Furthermore, the results only represent an 
average for the flare sample. Different flaring regions are not likely to have identical geometry. Nor are individual flares likely to have time independent electron distributions. Therefore, to do detailed studies of the particle distributions, one needs a technique that can measure time dependent anisotropies on a flare by flare basis. Polarization measurements are a diagnostic that meet these requirements.

There have been a number of measurements of flare X-ray polarization at low energies $(\sim 10 \mathrm{keV})$. The first flare measurements indicated polarization levels in the range of $20-40 \%(8,9)$. Although these reports were met with considerable skepticism (10), subsequent observations (11) confirmed the existence and magnitudes of the polarizations $(\sim 10 \%)$. Later studies measured polarizations of only a few percent at $\sim 15 \mathrm{keV}$ for two flares in July 1974 (12). The small but finite polarization is consistent with the predictions for purely thermal emission that contains an admixture of polarized backscattered radiation (13).

The thermal component, with its rather low polarization, tends to dominate the emission from all flares at energies below about $25 \mathrm{keV}$. In fact, the idea that the emission in this energy band is primarily thermal finds support from more recent polarization measurements (14). These data indicate that the polarization in the $5-25 \mathrm{keV}$ band tends to be less than $\sim 4 \%$, consistent with isotropic unpolarized emission that is slightly polarized by backscattering. It has been pointed out that, because of this thermal "contamination", effective polarization measurements can only be performed at higher energies (15).

At higher energies $(>100 \mathrm{keV})$, we expect large linear polarizations based on the anisotropy of the electrons. Linear polarizations of $20 \%$ (16) or even larger $(13,17)$ can be expected. Polarization measurements can be expected to provide additional information not only on the degree of isotropy, but also on the exact nature of the electron anisotropy. At present, there is no $\gamma$ ray experiment in operation whose major emphasis is the measurement of polarization.

\section{X-RAY ALBEDO POLARIMETRY}

A measurement of the linear polarization of a transient event (such as a solar flare) can, in principle, be made by measuring the angular distribution of the albedo flux, i.e., the source flux which is scattered from the Earth's atmosphere prior to reaching the detector. This concept is based on the properties of the Compton scattering of polarized radiation. In particular, this approach relies on the fact that, in the case of linearly polarized radiation, the scattered photon tends to be ejected at right angles to the electric field vector of the incident radiation (18). The atmosphere, as seen from an orbiting satellite, presents a wide range of possible scatter angles for a given source direction. The photon scatter angle will depend on look direction. Hence, the intensity distribution of the albedo flux will exhibit an angular distribution which will 
depend on the polarization properties of the source radiation.

For the case of an orbiting detector observing some cosmic source, the distribution of the scattered albedo flux across the top of the atmosphere will depend on three parameters: 1) the angular height of the source above the Earth's limb (i.e., the source-earth-detector geometry); 2) the level of linear polarization of the source flux; and 3) the orientation of the bulk polarization vector of the source flux. Therefore, if one knows the direction of the source, then the polarization properties can be determined from the study of the albedo flux.

To illustrate the dependence on the polarization parameters, consider a source of radiation which is observed at the local zenith. In this case, any asymmetries in the observed albedo flux will be due solely to the polarization properties of the incident source flux. These asymmetries will manifest themselves in terms of an azimuthal dependence of the observed albedo flux. For an unpolarized source, the albedo flux will be uniformly distributed in azimuth. For a linearly polarized source, however, the observed albedo flux will exhibit an asymmetry about the azimuthal direction. This asymmetry will take the form of $\operatorname{as}^{2} \theta$ dependence on the azimuthal angle. The magnitude of these variations represents a measure of the polarization fraction, while the plane defined by the azimuthal minima corresponds to the plane of linear polarization in the incident source flux. The largest dependence of albedo distribution on incident polarization occurs for geometries where the source is located well away from the zenith $\left(30^{\circ}-40^{\circ}\right)$. This allows for single scatters at angles near $90^{\circ}$ where the sensitivity of the Compton cross section to polarization peaks. In these geometries, there will be asymmetries introduced even in the case of an unpolarized source. For example, for events which are close to the Earth's limb, the higher probability of forward scattering will result in a limb-brightening effect in the general direction of the source. A second type of asymmetry will arise from the fact that, for events with a zenith angle in the range of $70^{\circ}-110^{\circ}$, not all of the visible atmosphere is exposed to direct source flux. A precise measurement of polarization will require that all of these effects be properly acounted for.

We have modeled this scattering process using Monte Carlo simulations, incorporating a version of GEANT which has been modified to handle polarized photons. A library of simulation results has been assembled for photon beams directed at various angles of incidence on a flat slab of (oxygen-nitrogen) atmosphere. These simulations include both polarized and unpolarized photon beams as well as both mono-energetic and power-law source spectra. The effects of multiple scattering are also properly accounted for. The atmosphere of the Earth can then be simulated by combining these data in such a way as to simulate the spherical atmosphere as a superposition of scattering sites, each oriented at various angles with respect to the observer. (This approach is required due to the scaling limitations.) In this way, we have effectively simulated the distribution of the scattered flux for a beam of photons incident on the top of the atmosphere. 


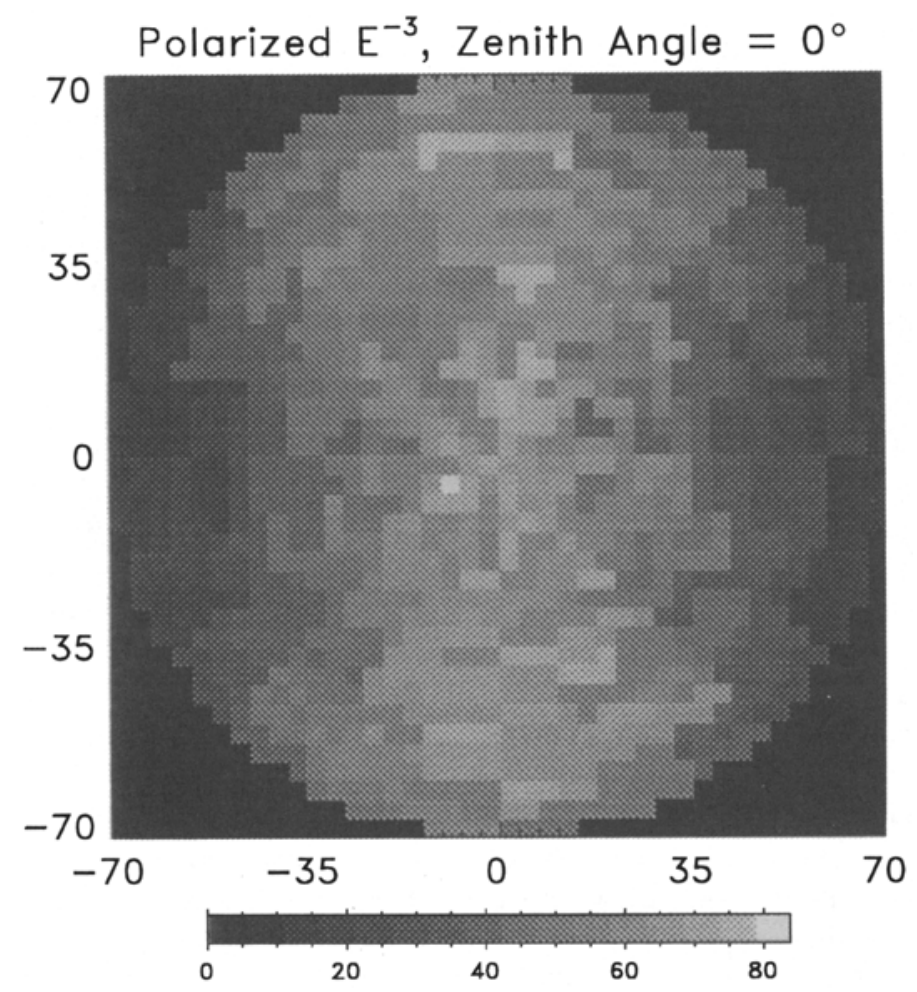

FIG. 1. The albedo distribution (as seen at the spacecraft) corresponding to a $100 \%$ polarized $\mathrm{E}^{-3}$ spectrum incident from $0^{\circ}$ zenith angle. The polarization (E-field) vector lies along the horizontal plane. The scale is a relative measure of the photon flux from each solid angle element.

For an incident $\mathrm{E}^{-3}$ power-law spectrum, the energy distribution of the scattered flux (integrated over $2 \pi$ steradian) peaks in the $50-100 \mathrm{keV}$ range. The vast majority of scattered photons in this energy range originate with photons of initial energy between 50 and $300 \mathrm{keV}$. For sources near the zenith, the albedo fraction reaches a maximum value of about $40 \%$. Examples of the spatial distribution of the scattered flux, based on our simulations, are shown in Figures 1-3. These figures show the intensity of the scattered flux across the visible disk of the Earth (which subtends an angle of $\sim 70^{\circ}$ in low Earth orbit) for the case of a $100 \%$ polarized source at zenith angles of $0^{\circ}, 30^{\circ}$, and $60^{\circ}$.

These simulations serve to demonstrate that, in principle, one could study solar flare polarization by mapping out the observed distribution of the flux 


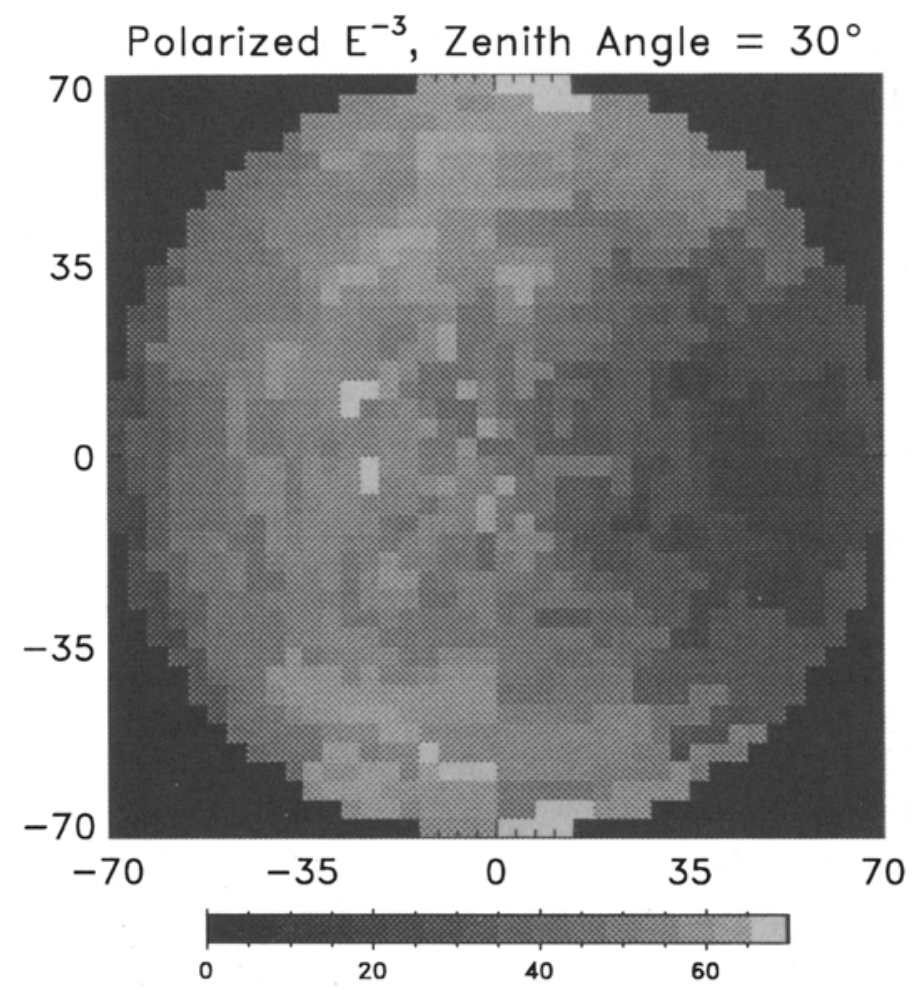

FIG. 2. The albedo distribution (as seen at the spacecraft) corresponding to a $100 \%$ polarized $\mathrm{E}^{-3}$ spectrum incident from $30^{\circ}$ zenith angle. The source lies in the $+x$ direction, with the polarization (E-field) vector lying along the horizontal plane. The scale is a relative measure of the photon flux from each solid angle element.

which is scattered off the atmosphere. A mapping of this distribution at a resolution of a few degrees would certainly provide sufficient detail for such an albedo polarimeter. Unfortunately, we have no way of mapping the albedo flux with such high precision at the present time. However, the BATSE detectors on CGRO (in particular, the Large Area Detectors, or LADs) are capable of providing a crude map of the distribution of the albedo flux. Despite the aliasing effects amongst the detectors, this capability is sufficient for measuring polarization parameters in some of the larger flare events. 


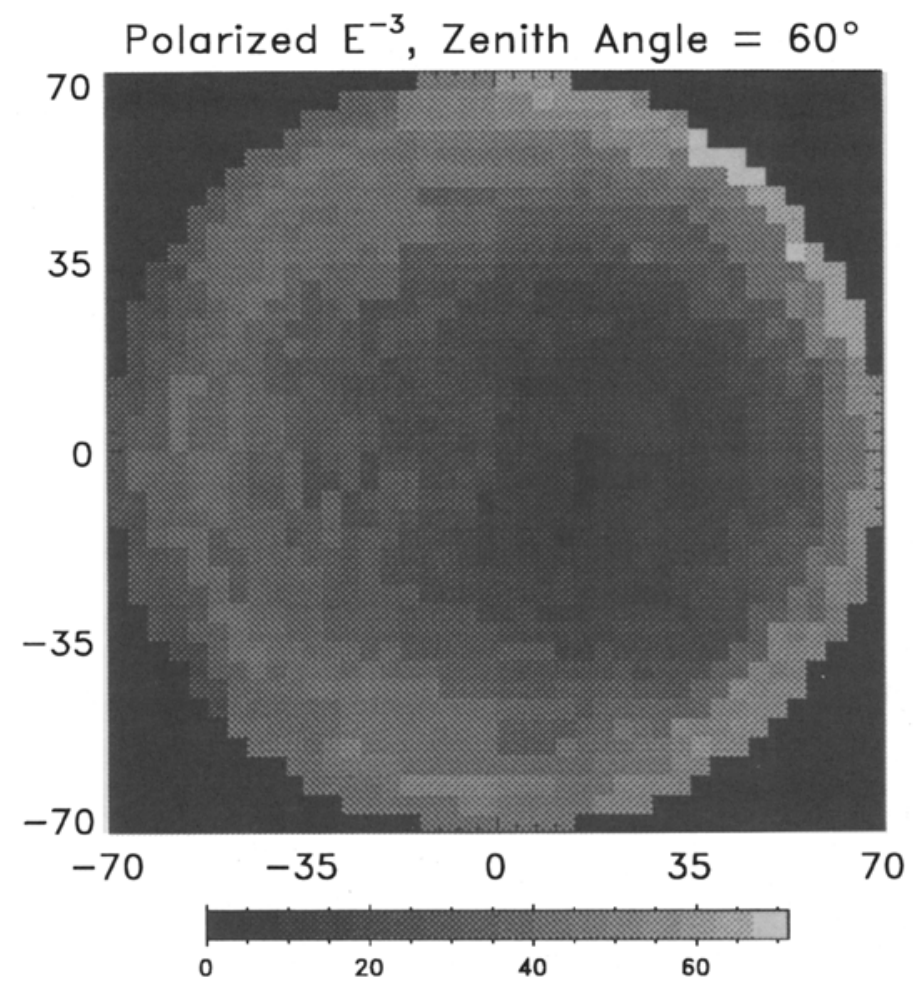

FIG. 3. The albedo distribution (as seen at the spacecraft) corresponding to a $100 \%$ polarized $\mathrm{E}^{-3}$ spectrum incident from $60^{\circ}$ zenith angle. The source lies in the $+x$ direction, with the polarization (E-field) vector lying along the horizontal plane. The scale is a relative measure of the photon flux from each solid angle element.

\section{BATSE SENSITIVITY TO FLARE POLARIZATION}

The exact sensitivity of the BATSE to the measurement of flare polarization depends on several factors. These include the following:

1) Source zenith angle. The sensitivity will depend on the contrast amongst the different LAD detectors. This depends not only on the contrast within the polarized flux distribution, but also on the contrast (or lack thereof) in the corresponding unpolarized distribution. These effects are such that the sensitivity is near maximum for source zenith angles $\sim 30^{\circ}$. 
2) Aliasing. Due to the large FoV and the geometrical spacing of the LAD detectors, there exist certain geometrical arrangements with respect to the polarization vector where the polarization sensitivity will be limited (and perhaps even go to zero). In more general terms, the polarization sensitivity will vary within the polarization parameter space.

3) Incident source spectrum. Because the scattered albedo photons tend to result from incident photons $>100 \mathrm{keV}$, a harder incident spectrum will result in more intense albedo flux and, hence, higher sensitivity. This means, for example, that there will be less sensitivity to a (typically softer) solar flare than to a (typically harder) $\gamma$-ray burst of comparable fluence.

4) Source inensity. For more intense events, various instrumental effects, most notably pulse pile-up and deadtime, become important factors.

5) Flux leakage. We have assumed an LAD angular response which goes to zero at $90^{\circ}$. In other words, we assumed that the leakage of flux through the spacecraft can be neglected. (This is known not to be the case for some of the larger events and may even be the limiting factor in the application of this technique to BATSE data.)

6) Background level. The total background level for a given event depends not only on the background rate, but also on the duration of the event.

In estimating BATSE sensitivity levels, we consider only the four detectors which face away from the sun and which are exposed only to the albedo flux. We then search for a distribution of events in those detectors which are statistically inconsistent with that expected from an unpolarized event. In practice, this involves a $\chi^{2}$ comparison between the event distribution derived from albedo simulations for a polarized and an unpolarized solar flare at a given zenith angle.

In order to relate our sensitivity estimates to typical BATSE solar flare events, we chose three events on the basis of zenith angle $\left(<45^{\circ}\right)$, duration ( $<5$ minutes) and intensity. Our simulation data was then used to determine the minimum level of polarization which could be observed in each case. The results are shown in Table 1 . These results suggest that this technique is capable of reaching reasonable sensitivity levels $(\sim 20 \%)$ for modest-sized

solar flares. For the larger events, sensitivity levels below $10 \%$ should be achievable.

\section{BATSE MEASUREMENTS OF FLARE POLARIZATION}

At the present time, we have no firm results (either positive detections or upper limits) for any solar flare observed by BATSE. The analysis is presently 
TABLE 1. Estimated polarization sensitivity levels for three typical BATSE solar flare events. The sensitivity levels reflect the percent polarization required for a $3 \sigma$ detection.

\begin{tabular}{ccccccc}
\hline $\begin{array}{c}\text { Trigger } \\
\text { No. }\end{array}$ & Date & Class & $\begin{array}{c}\text { Zenith } \\
\text { Angle }\end{array}$ & $\begin{array}{c}\Delta t \\
(\mathrm{secs})\end{array}$ & $\begin{array}{c}\text { Total } \\
\text { Counts }\end{array}$ & $\begin{array}{c}\text { Sensitivity } \\
(3 \sigma)\end{array}$ \\
\hline 629 & 5-Aug-91 & M1.2 & $45^{\circ}$ & 75 & 252,000 & $40 \%$ \\
688 & 16-Aug-91 & C9.6 & $18^{\circ}$ & 75 & 694,000 & $35 \%$ \\
1032 & 9 -Nov-91 & M1.4 & $43^{\circ}$ & 100 & 975,000 & $20 \%$ \\
\hline
\end{tabular}

dominated by several systematic effects which preclude any definitive measurements. These effects include: 1) differences in channel bandwidths amongst the LAD detectors; 2) deadtime effects; 3) leakage of incident flux through the spacecraft; and 4) the need to account for the precise form of the incident spectrum. The ongoing analysis is concentrating on the effort to remove these systematics effects. We are also in the process of setting up our simulation database in a form that can be directly used by software tools developed by the BATSE team. This should greatly facilitate the search for solar flare polarization in the BATSE database.

\section{ACKNOWLEDGEMENTS}

This work is supported at UNH by NASA Grant NAG 5-2388 and by NASA contract NAS 5-26645.

\section{REFERENCES}

1. Vestrand, W. T., et al., Ap. I. 322, 1010 (1987).

2. Bogovlov, S.V., et al., Soviet Astr. Letters, 11, 322 (1985).

3. Catalano, C.P. and Van Allen, J.A., Ap. J., 185, 335 (1973).

4. Kane, S.R., et al., Ap. J. Lett., 254, L53 (1982).

5. Vestrand, W. T. and Ghosh, A., 20th ICRC, 3, 57 (1987)

6. Mctiernan, and Petrosian, V., Ap. J., 359, 541 (1990).

7. Petrosian, V., Ap. J., 299, 987 (1985).

8. Tindo, I.P., et al., Sol. Phys., 14, 204 (1970).

9. Tindo, I.P., et al., Sol. Phys., 27, 426 (1972).

10. Brown, J.C., McClymont, A.N. and McLean, I.S., Nature, 247, 448 (1974)

11. Nakada, M.P., Neupert, W.M. and Thomas, R.J., Sol. Phys., 37, 429 (1974).

12. Tindo, I.P., Shuryghin, A.I. and Steffen, W., Sol. Phys., 46, 219 (1976).

13. Bai, T. and Ramaty, R., Ap. J., 219, 705-726 (1978).

14. Tramiel, L.J., Channan, G.A. and Novick, R., Ap. J., 280, 440 (1984).

15. Chanan, G.A., Emslie, A.G. and Novick, R., Sol. Phys., 118, 309 (1988).

16. Emslie, A.G. and Vlahos, L., Ap. J., 242, 359 (1980).

17. Leach, J. and Petrosian, V., Ap. J. Lett., 269, 715 (1983).

18. Evans, R.D. The Atomic Nucleus, New York: McGraw-Hill Book Co. (1955). 